\title{
Short-term recognition memory for serial order and timing
}

\author{
Simon Farrell and Karis McLaughlin \\ University of Bristol, Bristol, England
}

\begin{abstract}
Recent evidence suggests that a common temporal representation underlies memory for serial order of items in a sequence, and the timing of items in a sequence. This stands in contrast to other data suggesting a reliance on only ordinal information in short-term memory tasks. An experiment is reported here in which participants were post cued to perform a comparison between a probe and study list of items irregularly spaced in time, on the basis of order or temporal information. Participants' performance on the serial recognition task was not affected by the temporal proximity of items, although participants were able to use temporal information to perform a temporal recognition task. Application of a temporal matching model of serial and temporal recognition suggests that although participants were able to remember the timing of items, this memory for timing was unlikely to determine serial recognition performance. The results suggest a dissociation between ordinal and temporal information in short-term memory.
\end{abstract}

Numerous contemporary models of memory are dedicated to explaining short-term order memory, and in doing so adopt a variety of assumptions to explain people's ability to maintain and recall the order of items or events in a sequence over the short term. In some models, such as chaining models (e.g., Lewandowsky \& Murdock, 1989; Murdock, 1995) and ordinal models (e.g., Farrell \& Lewandowsky, 2002; Page \& Norris, 1998), ordering is assumed to follow from the relationships between items (associations and relative strengths, respectively). In another class of models, the ordering of items is assumed to follow from the pairing of items with some external representation of the location of an item in a sequence. Of interest here is a subset of these models that assume that the order of items is represented by placing them along a temporal dimension. In temporal context models, such as the connectionist OSCAR model of Brown, Preece, and Hulme (2000), items are ordered by associating each item with a vector representing temporal context; being the output of a bank of sinusoidal oscillators, this temporal context evolves over time and can be used to specify the position of an item in a sequence (see also Burgess \& Hitch, 1999). In a similar spirit, temporal distinctiveness models take a more abstract approach to list memory, and treat recall as the discrimination of items along a temporal dimension (e.g., Brown, Neath, \& Chater, 2007; Glenberg \& Swanson, 1986; Neath, 1993). Although differing in level and details of implementation, these time-based models ${ }^{1}$ have in common the assumption that memory for the order of events follows from (temporal context models) or is primarily affected by (temporal distinctiveness models) memory for the timing of those events.
A fundamental feature of time-based models is that they predict effects of the temporal isolation of items on ordered recall accuracy. Specifically, the more an item is separated in time from nearby items on a memory list, the more accurately that item is predicted to be recalled. In the case of OSCAR (Brown et al., 2000), cross-talk in the connectionist network that stores the associations between temporal context and items in a sequence means that items that are stored in more similar temporal contexts will interfere more with each other at retrieval when one of those temporal contexts is used as a cue. Temporal distinctiveness models predict this temporal isolation effect (TIE) by virtue of decreased temporal distinctiveness of the items. For example, the recent SIMPLE (scale-invariant memory, perception, and learning) model treats time as a dimension along which items may be discriminated, just like perceptual dimensions such as weight or brightness (Brown et al., 2007; Neath \& Brown, 2006). For a demonstration of the prediction of a TIE in SIMPLE, see Figure 1 of Lewandowsky, Brown, Wright, and Nimmo (2006).

A number of experiments have recently been published that systematically call into question these time-based models by demonstrating a lack of TIEs in short-term memory for order. The general methodology employed in these experiments has been to present participants with items irregularly spaced in time, and to examine any effects of the temporal interval preceding and following an item on the accuracy of its serial recall. Across a wide variety of manipulations such as modality of list presentation (auditory and visual presentation: Nimmo \& Lewandowsky, 2006; Parmentier, King, \& Dennis, 2006), varying temporal intervals over different time scales (Nimmo

S. Farrell, simon.farrell@bristol.ac.uk 
EXAMPLE LIST

1

79

5

Time in sequence

SERIAL RECOGNITION PROBES

\begin{tabular}{|llllll|}
\hline identical & 1 & 3 & 7 & 9 & 5 \\
close lure & 1 & 3 & 9 & 7 & 5 \\
far lure & 1 & 7 & 3 & 9 & 5 \\
\hline
\end{tabular}

TEMPORAL RECOGNITION PROBES

\begin{tabular}{lllll|}
\hline identical & 1 & 3 & 7 & 9 \\
close lure & 1 & 3 & 7 & 5 \\
far lure & 1 & 3 & 5 & 5 \\
\hline
\end{tabular}

Figure 1. Trial structure, indicating possible probe types for serial recognition trials and temporal recognition trials. See text for a description.

\& Lewandowsky, 2005), and use of different order recall procedures (serial recall and partial-report probed recall; see, e.g., Lewandowsky et al., 2006), there has been a consistent failure to observe the TIEs predicted by time-based models of short-term order memory. One exception is an initial experiment conducted by Lewandowsky and Brown (2005), who observed that longer intervals following an item were associated with more accurate serial recall of such items, consistent with time-based models. However, Lewandowsky and Brown noted that such a result is also consistent with the time following an item being used to employ encoding strategies such as grouping or chunking (see Ryan, 1969), or being used for consolidation. Notably, when procedures such as articulatory suppression have been used to minimize the employment of encoding or rehearsal strategies on the part of participants, a TIE in forward order recall has never been observed (Lewandowsky \& Brown, 2005; Lewandowsky et al., 2006; Nimmo \& Lewandowsky, 2005).

The unanimous demonstration of a lack of effect of temporal isolation on short-term memory for order when encoding strategies are controlled would appear to mitigate against time-based models of short-term order memory. Nevertheless, there are other considerations that suggest that the role of temporal information in short-term memory requires further investigation. One factor is the sharp contrast between the TIE results for serial recall, and other experiments that have varied task demands. Brown, Morin, and Lewandowsky (2006) replicated the temporal isolation experiments in free recall using an unpredictable temporal structure as in the serial recall experiments con- ducted by Lewandowsky and colleagues. Strikingly, and consistent with experiments employing a predictable temporal schedule, a TIE emerged: Temporally isolated items were more accurately freely recalled than those items that were temporally crowded. Lewandowsky, Nimmo, and Brown (in press) provided further evidence showing that temporal isolation effects may also be observed in order memory when output order is unconstrained. They used a reconstruction-of-order task and found a TIE when participants could provide the position of items in any output order, but failed to find a TIE when output order was forced to match input order. In contrast to serial recall, these findings support temporal distinctiveness theories, which have been particularly concerned with the link between temporal scheduling and recency effects in free recall (e.g., Bjork \& Whitten, 1974; Glenberg, 1987; Neath, 1993; Neath \& Crowder, 1990).

More direct evidence for the role of temporal information in short-term memory comes from experiments that specifically require the recall of the temporal schedule of items (Collier \& Logan, 2000; Crowder \& Greene, 1987; Elvevåg, Brown, McCormack, Vousden, \& Goldberg, 2004; Glenberg \& Jona, 1991; Saito, 2001; Watkins, LeCompte, Elliott, \& Fish, 1992). For example, Watkins et al. presented participants with irregularly timed sequences of signals such as beeps, flashes and digits, and had participants reproduce the timing of the items. Watkins et al. found that participants ably performed the task, and also found that an expected effect of modality of presentation (auditory or visual) was only present under certain conditions. Similarly, Crowder and Greene presented participants with sequences of letters, irregularly spaced in time, for study. At test, participants were presented with two successive pairs of items, and were asked to judge which of the two intervals was longer on the study list. Across a range of manipulations, Crowder and Greene found that participants performed well on this temporal discrimination task. Crowder and Greene, along with others (see, e.g., Watkins et al., 1992) concluded that memory for the timing of items can and should be distinguished from memory for their order, and is worthy of study in its own right.

Intriguingly, some investigations of the memory for timing have additionally observed a relationship between memory for timing and memory for order, an observation that is inconsistent with the TIE data outlined earlier. For example, Saito (2001) observed that, across individuals, digit span scores were positively correlated with shortterm memory for rhythmic patterns; that this relationship remained even after partialling out of common variance due to reading speed suggests a specific relationship between memory for time and order (Saito, 2001). Consistent with this suggestion, Elvevåg et al. (2004) found that patients with schizophrenia were impaired, relative to controls, on both a timing task (duration identification) and a short-term order memory task (probed recall). Importantly, these deficits did not extend to judgments of line length, a visual analogue of the timing task, suggesting a common timing deficit underlying the impaired performance on the timing and order tasks in these patients. In 
contrast to the conclusions from the TIE literature, these findings are consistent with the notion that serial order memory does rely on some form of timing information.

This leaves us with the question: Are memory for time and memory for order simply different faces of the same representational coin, or is it possible to dissociate time and order memory? According to Brown et al. (2006), the findings from the TIE literature suggest that people may rely on a temporal dimension or an ordinal (i.e., serial position) dimension to discriminate between items in memory experiments. On the other hand, the results of Saito (2001) and Elvevåg et al. (2004) suggest a common basis for memory for timing and order. This paper reports and experiment and some model simulations that place constraints on theories of the relationship between timing and order in short-term memory. Participants were presented with list items and asked to pay attention to the order of the items, and their timing; after presentation of list items the participants were instructed to perform a recognition task on the basis of the order of items (i.e., serial recognition), or their timing (i.e., temporal recognition; see Collier \& Logan, 2000; Ross \& Houtsma, 1994). Assuming a common temporal dimension for timing and order memory, the experiment provides conditions favorable for observing TIEs in serial order memory by explicitly requiring that participants attend to the timing of items. Second, in the framework of hybrid temporal-ordinal models, postcuing allows one to determine whether the importance given to temporal and ordinal dimensions in serial order recall occurs at encoding (by selectively attending to and encoding temporal or ordinal information) or at retrieval (via the attentional weighting of dimensions). Third, the experiment adds to the relatively sparse literature on short-term memory for the timing of sequences.

\section{EXPERIMENT}

The experiment was designed to assess time-based models of short-term memory by changing the timing of items (see, e.g., Lewandowsky \& Brown, 2005; Nimmo \& Lewandowsky, 2005). Participants were presented with digits quasirandomly scheduled in time, and were then postcued to perform serial recognition (are the items in the probe sequence in the same order as the presented list?) or temporal recognition (do the items in the probe sequence have the same timing as those in the presented list, disregarding their order?). In the serial recognition task, lures were identical to presented lists with the exception that two adjacent items were swapped; critically, the two swapped items could be closely or widely separated in time (see Figure 1). This task provides a strong test of models in which order memory is reliant only on memory for the times of items; these models predict a higher false recognition rate for lures swapping two close items (compared to two widely separated items). The temporal recognition task provides useful data on short-term memory for time; since time-based models assume that participants memory for the time of events is used to determine their order, a necessary demonstration is that people can remember the timing of events over the short term (cf. Collier \& Logan,
2000; Crowder \& Greene, 1987; Pich, 2000; Saito, 2001; Watkins et al., 1992), and that these data are not explainable within the framework of order-based models.

\section{Method}

Participants and Design. Participants were 30 undergraduate students from the University of Bristol aged 20-24 years $(M=21$, $S D=0.85$ ), who were recruited as part of an opportunity sample, and provided informed consent for participation. All participants were native English speakers. Each participant completed trials in all cells of the 2 (task type: order vs. timing) $\times 3$ (recognition probe: same, close, and far) design.

Materials and Apparatus. The items presented on memory lists and probe sequences were the digits 1-9, the digits being presented auditorily to participants. Although the evidence for a modality effect in memory for timing is ambiguous, the partial evidence in favor of an auditory superiority effect led us to use auditory presentation (see, e.g., Collier \& Logan, 2000; Glenberg \& Jona, 1991; Glenberg, Mann, Altman, Forman, \& Procise, 1989; Glenberg \& Swanson, 1986; but see Nimmo \& Lewandowsky, 2006; Schab \& Crowder, 1989; Watkins et al., 1992). The spoken numbers were recorded from a male speaker with a neutral English accent, and digitised for storage on computer. The digits were pitch-flattened to a fundamental frequency of $113 \mathrm{~Hz}$ using the phonetics software Praat (Boersma \& Weenink, 2006) - to avoid any grouping effects arising from pitch variations (see Nicholls \& Jones, 2002) - and normalized to a constant length of $500 \mathrm{msec}$. The digits were additionally padded to give a regular sounding sequence for different permutations, as it is known (and was observed in pilot testing) that the subjective experience of the center of an acoustic stimulus does not exactly relate to the onset of the stimulus (e.g., Scott, 1998).

A study list was constructed for each trial by randomly sampling five digits, without replacement, from the digits $1-9$. The silent pauses between the digits on each list were random permutations of the set of pauses $\{200 \mathrm{msec}, 500 \mathrm{msec}, 1,000 \mathrm{msec}, 2,200 \mathrm{msec}\}$. The probe for each trial was constructed according to the task (order or timing) and the nature of the probe. For both order and timing tasks, same probes were identical to the corresponding input lists, both in the identity and order of the digits, and in their timing. For the serial recognition task, two different types of lures, close and far, were constructed by swapping two adjacent digits. For close lures, the two items swapped were those adjacent digits separated by the smallest pause $(200 \mathrm{msec})$ on the input lists. For far trials, the two adjacent digits separated by the longest pause $(2,200 \mathrm{msec})$ were swapped (see Figure 1). The timing of the close and far lures for the serial recognition task was identical to that of the study lists. Two types of lures were also constructed for the temporal recognition task. In this case, the identity and order of the items remained constant, and only their timing was changed: On close trials, the two most similar adjacent pauses were swapped, while on far trials, the two most different adjacent pauses were swapped (see bottom part of Figure 1). In total each participant received 40 order trials and 40 rhythm trials; within each task type, participants received 20 same probes, 10 close probes, and 10 far probes. Six practice lists were also constructed ( 3 order trials and 3 rhythm trials, 1 for each type of recognition probe).

The experiment was run on a PC, with the Psychophysics Toolbox for MATLAB (Brainard, 1997; Pelli, 1997) being used to control stimuli presentation and collect participants' responses.

Procedure. Participants were tested individually in a secluded room. A fixation point was presented centrally on the screen for $1,000 \mathrm{msec}$ to mark the beginning of a trial. A $1,000-\mathrm{msec}$ blank screen followed, which was then succeeded by presentation of the memory list over the headphones. During presentation of the list, rehearsal was prevented by articulatory suppression: Participants were required to repeatedly vocalise the word "sugar" out loud, beginning at the presentation of the fixation cross, and ending with the presentation of the task cue. The task cue, either NUMBERS (indicating that participants should make their same/different decision on the basis 
Order Task

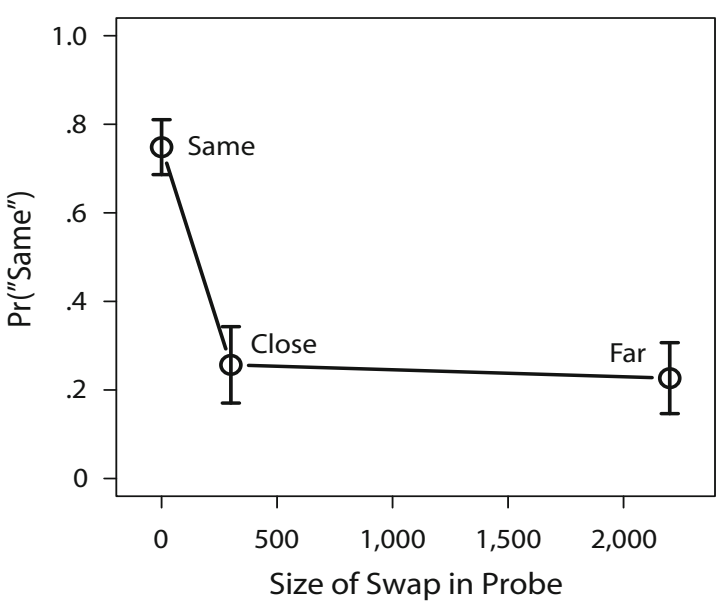

Timing Task

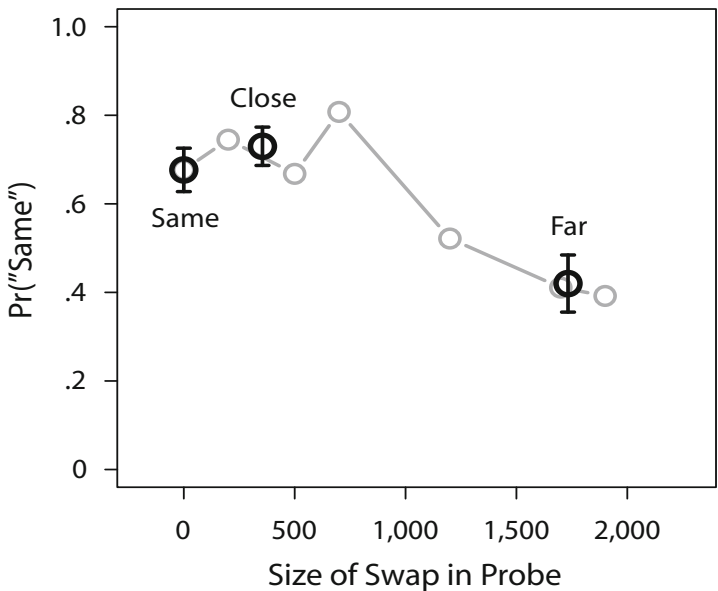

Figure 2. Proportion "same" responses for the serial recognition task (left panel) and the temporal recognition task (right panel). For the serial recognition task, performance is plotted as a function of the interval separating the two swapped items. For the temporal recognition task, performance is plotted as a function of the size of the displacement, in time, of the item perturbed to make the probe. Data for the temporal recognition task are grouped according to the displacement in time of the item perturbed in the lure (gray), and also by the condition (black symbols). Error bars for condition means in all figures depict repeated measures confidence intervals (Bakeman \& McArthur, 1996).

of the order of the digits) or RHYTHM (indicating that participants should make their same/different decision on the basis of the timing of the digits) was presented centrally on the screen for $1,000 \mathrm{msec}$, followed by presentation of the recognition probe over the headphones. After presentation of the probe sequence the recognition prompt DIFFERENT OR SAME was displayed centrally for $1,000 \mathrm{msec}$, upon which the participant was required to press the " $z$ " key if they thought the second list was different to the first list (on the basis of the information indicated by the task cue), or the "?" key to indicate that the two lists were the same. Participants were blocked from providing a response until this response screen appeared. A 1,000-msec blank screen intertrial interval preceded commencement of the next trial. The trials were continuous, with each consecutive trial being triggered by the participant's response to the previous trial. Selfpaced breaks were allowed every 20 trials, in which the participant pressed a computer key to continue with the experiment, instigating a 2,000-msec pause before commencement of the next trial. Each testing session lasted approximately $40 \mathrm{~min}$. Six practice trials were presented before the experimental session began ( 3 order trials and 3 rhythm trials, 1 for each type of recognition probe), in order to familiarize participants with the experiment.

\section{Results}

Serial recognition. The mean proportion of "same" responses for the three probes in both tasks is plotted as a function of the interval separating the swapped items in Figure 2. Looking first at the results for the serial recognition task (left panel), it is clear that participants were overall able to discriminate between identical and lure probes: A one-way repeated measures $\mathrm{ANOVA}^{2}$ conducted on the proportion "same" responses revealed a significant effect of probe type $\left[F(1.45,42.23)=69.63 ; M S_{\mathrm{e}}=0.051 ; p<\right.$ $.001]$. Post hoc $t$ tests revealed significant differences in the proportion of "same" responses to same and close probes $[t(29)=8.47, p<.001]$. In contrast, no significant difference in "same" responses was obtained between close and far lures $[t(29)=0.96, p=.34]$.
Signal detection analyses were run to confirm that this pattern of results was primarily related to the discriminability of distributions. A mixed-effects logistic regression model was fit to the data using the glmmPQL package in the statistics software R (available from www.R-project .org). Logistic regression has previously been offered as a useful method, available in standard statistics packages, for estimating signal detection parameters (see, e.g., DeCarlo, 2003). Mixed effects models are a preferable alternative to analyses on individuals' parameter estimates (e.g., by estimating parameters for individuals and then submitting these to analysis by ANOVA) in treating the effects of individuals as random, not fixed, effects (Pinheiro \& Bates, 2000). A major advantage of this is "shrinkage": Extreme parameter estimates for individuals are avoided by weighting individual parameter estimates by a constraining parent distribution (similar to Bayesian multilevel signal detection procedure demonstrated by Rouder \& Lu, 2005). Using logistic regression with a probit link, the slopes are directly interpretable as estimates of $d^{\prime}$, while the intercept estimates the criterion $-c$. Two hierarchical regressions were performed; one comparing "same" and "close" response rates, the other performed on "close" and "far" response rates. The estimated $d$ ' for the same versus close comparison (1.51) differed significantly from $0[t(869)=7.23, p<.001]$, but the mean $d^{\prime}$ for the close versus far comparison (0.09) did not reach significance $[t(569)=0.80, p=.42]$.

Temporal recognition. The mean "same" response proportions for the temporal recognition task are shown in the right panel of Figure 2. The gray symbols plot the data by the individual sizes of swaps in the experiment (there were 6 possible swap sizes in the close and far conditions; the swap size of 0 corresponds to the same condition). Not 
all participants contribute to each mean since the exact set of swap sizes received by each participant was randomized; these data are therefore not amenable to analysis, but are suggestive of a systematic underlying function that is flatter for smaller swap sizes, and more steeply sloped for larger swap sizes. The black symbols show the results plotted by condition as in the left panel. A repeated measures ANOVA revealed overall effects of probe type on "same" response proportions $[F(1.65,47.81)=27.18$, $\left.M S_{\mathrm{e}}=0.037, p<.001\right]$. Post hoc $t$ tests revealed that "same" response proportions did not differ significantly between same and close probes $[t(29)=-1.60, p=.12]$; however, a significant difference was observed between close and far lures $[t(29)=6.49, p<.001]$.

A hierarchical signal detection analysis was performed as for the serial recognition data. The mean estimated $d^{\prime}$ for the same versus close comparison $(-0.14)$ did not differ significantly from $0[t(869)=-1.40, p=.16]$; a significant difference from 0 was found for the estimated $d^{\prime}$ for the close versus far comparison $(0.84)[t(569)=6.32$, $p<.001]$.

\section{Discussion}

Participants could discriminate between same and close probes, but not close and far probes, in the serial recognition task. In contrast, for the temporal recognition task participants did not discriminate between same and close probes, but could ably distinguish between close and far probes. The results suggest that people do not perform serial recognition decisions on the basis of temporal information, and thus provide further constraints on time-based models of short-term order memory. Whereas pure time-based models predict that the temporal displacement of items on lures should affect the probability of identifying such lures as being the same as the input sequence on a serial recognition task, no such differences were observed in the experiment. In the framework of models incorporating both temporal and positional information, the results suggest that participants can selectively attend to temporal or positional information, and that this attention can be allocated at the time of test (see Lewandowsky et al., in press). We return to these conclusions after considering a computational model of the serial and temporal recognition tasks.

\section{MODELING}

To further explore the relationship between the assumed representations of time-based models and the results from the experiment, quantitative modeling of the data was desired. An examination of the literature shows that models of short-term order memory, including time-based models such as OSCAR and SIMPLE, have not been applied to the serial recognition task. In particular, although the time-based SIMPLE model provides a framework for representing the timing of items, it does not straightforwardly account for serial or temporal recognition. This is because the model is only concerned with the relative distance (i.e., match) of items in the past from the present; thus, SIMPLE cannot take into account the match between temporally extended study and test probes in an obvious fashion. Additionally, although there exist formal models of timing in the literature (e.g., Gallistel \& Gibbon, 2000; Staddon \& Higa, 1999; Wearden \& Doherty, 1995), these have no application to the memory for the timing or order of sequences of events. Accordingly, a unidimensional matching model of serial and temporal recognition is introduced here. In the model, forgetting is attributed to perturbation of temporal information over time. The model is described below and applied to the data from the order task and the timing task. The model is compared to a version which relies on positional, rather than temporal, information to represent the order and timing of events.

\section{Temporal Matching Model of Serial and Temporal Recognition}

The model is based on principles found in perturbation theories applied to memory for perceptual and ordinal information (Estes, 1997; Flexser \& Bower, 1974; Lee \& Estes, 1977), and Ratcliff's (1981) theory of perceptual matching; a schematic of the model is shown in Figure 3. First, it is assumed that events in a sequence are arrayed along a linear temporal dimension. ${ }^{3}$ Second, it is assumed that the time between study and test introduces uncertainty about the temporal placement of items on the study list; this uncertainty is assumed to be primarily due to perturbation, between study and test, of the representation of the temporal placement of items (see Lee \& Estes, 1977, and Estes, 1997, for a consideration of perturbation processes applied to loss of order and perceptual information, respectively), although encoding (Ratcliff, 1981) and retrieval (Flexser \& Bower, 1974) processes may also introduce noise. This uncertainty is implemented as the addition of normally distributed noise to the temporal placement of items in input sequences; this noise is assumed to have a mean of 0 and a standard deviation $\sigma$.

At test it is assumed that the noise-free probe sequence is matched to the noisy internal representation of the study sequence; this matching process is assumed to differ between serial and temporal recognition. In the case of serial recognition, the difference between the placement of an item at study and placement of the same item on the probe sequence is calculated (where placement is determined with respect to beginning of the sequence; see Figure 3); these distances are then summed across items to give an overall measure of the disparity between the probe sequence and the remembered study sequence. A similar distance calculation is assumed for temporal recognition; however, for temporal recognition it is assumed that the identity of items is ignored. Thus, an item on the probe sequence is not matched to the same item on the input sequence, but is rather matched to the item at the same position on the input sequence (see Figure 3). Finally, the summed distance measure for either task is compared to a criterion; if the overall calculated distance exceeds the criterion a "different" response is made, otherwise a "same" response is made. Separate criteria $\mathrm{C}_{\mathrm{S}}$ and $\mathrm{C}_{\mathrm{T}}$ were assumed for the serial recognition task and temporal recognition task (where $\mathrm{C}_{\mathrm{S}}$ and $\mathrm{C}_{\mathrm{T}}$ are free parameters).

This framework allows comparison of the predictions of time-based models and ordinal models. In time-based 


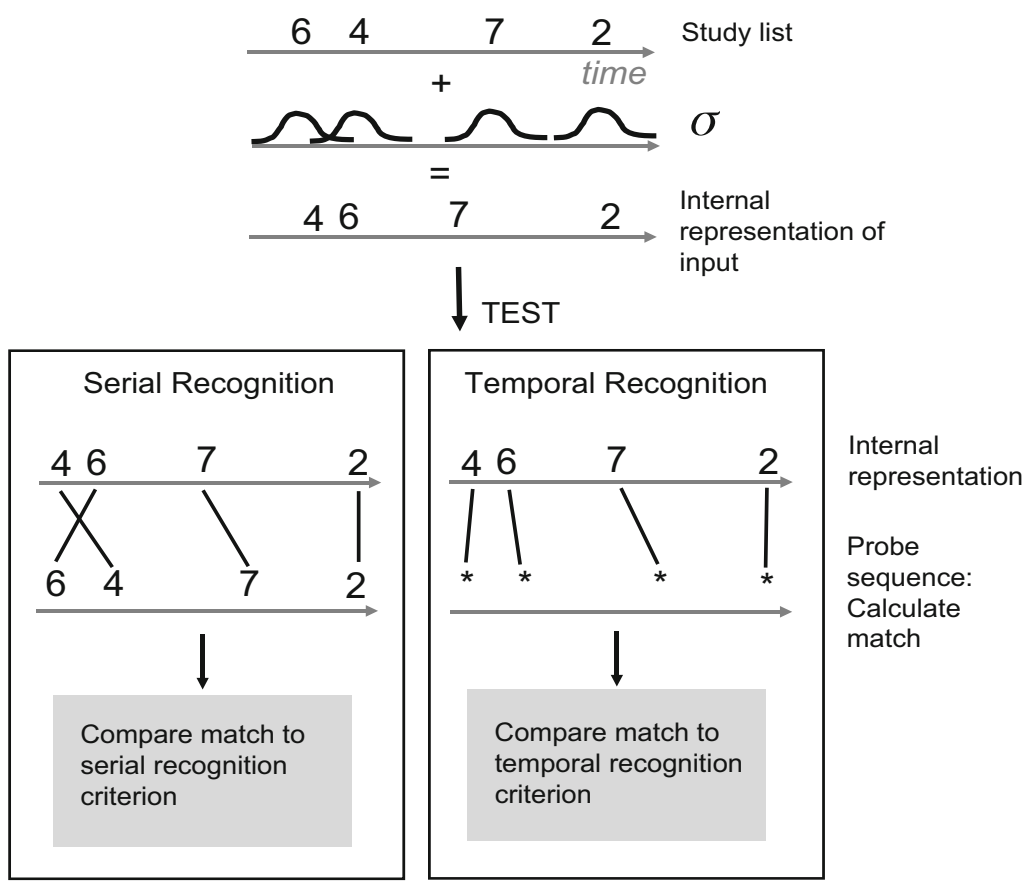

Figure 3. Matching model of serial and temporal recognition. The internal representation of the study list is assumed to be a temporal array of items perturbed by normally distributed noise. The internal representation is then matched to the probe sequence regarding (serial recognition) or disregarding (temporal recognition) the identity of items, the match being then compared to a criterion to produce a "same" or "different" response.

models, the temporal dimension is assumed to directly encode the timing of the input sequence. In contrast, in ordinal models any timing information is removed, with only the information about the order of the items remaining (see Lee \& Estes, 1977; Neath, 1999). For example, in the temporal model three items might be located at $300 \mathrm{msec}$, $900 \mathrm{msec}$ and $1300 \mathrm{msec}$ along the temporal dimension; in the order-based version of the model the same items would have values of 1,2 , and 3 along the (scale-free) positional dimension. In the simulations now reported, the ability of these two types of representation to account for performance in the two tasks is examined.

\section{Simulations}

The models were fit to the serial recognition and timing recognition data separately. To allow comparisons across models (where the temporal dimension was in msec units, while the positional model increased in steps of 1 ), the "time line" on each trial was standardized to a length of 1 (by dividing each item's presentation time by the constant total time of each list given the assumed dimension, temporal or positional). Each model incorporated two free parameters for each task. One was the criterion $\left(\mathrm{C}_{\mathrm{S}}\right.$ or $\left.\mathrm{C}_{\mathrm{T}}\right)$ placed on the amount of evidence to determine the "same" or "different" response. Rather than allowing the noise parameter $\sigma$ to freely vary (which introduced problems with negative variances in some of fits for individual participants), we instead assumed that the noise was drawn from a standardized normal distribution $(\mu=0, \sigma=1)$, and the second parameter was then the weighting given to the single positional or temporal dimension, $w_{\mathrm{D}}$.

Best-fitting parameter values were estimated for each participant using the SIMPLEX routine (Nelder \& Mead, 1965), minimizing the negative $\log$ likelihood $(-\ln L)$ of the data given the model. Predictions on each iteration of the SIMPLEX algorithm were calculated from 1,000 runs of the model, where each run used all 24 permutations of ISIs. A probability $p_{\text {same }}$ of responding "same" was calculated from the resulting 24,000 simulated trials, and a likelihood was then determined under a binomial distribution with probability $p_{\text {same }}(N$ being determined by the number of trials an individual participant received in that condition: 20 for same trials, and 10 each for close and far trials). For each participant, the SIMPLEX routine was run 25 times, each run of the fitting routine being given different starting values drawn from a grid covering a wide range of reasonable parameter values. The final fit for a participant was then the best fit obtained across the 25 SIMPLEX runs.

The simulation results for the serial recognition task are shown in the left panel of Figure 4, which shows the "same" response proportions predicted by the two models, averaged across individual participants. Figure 4 shows that although the temporal model could produce the appropriate difference in "same" response proportions between the same and close probes, the model over-predicts the difference between the close and far lures. To predict no difference between close and far lures the model would 

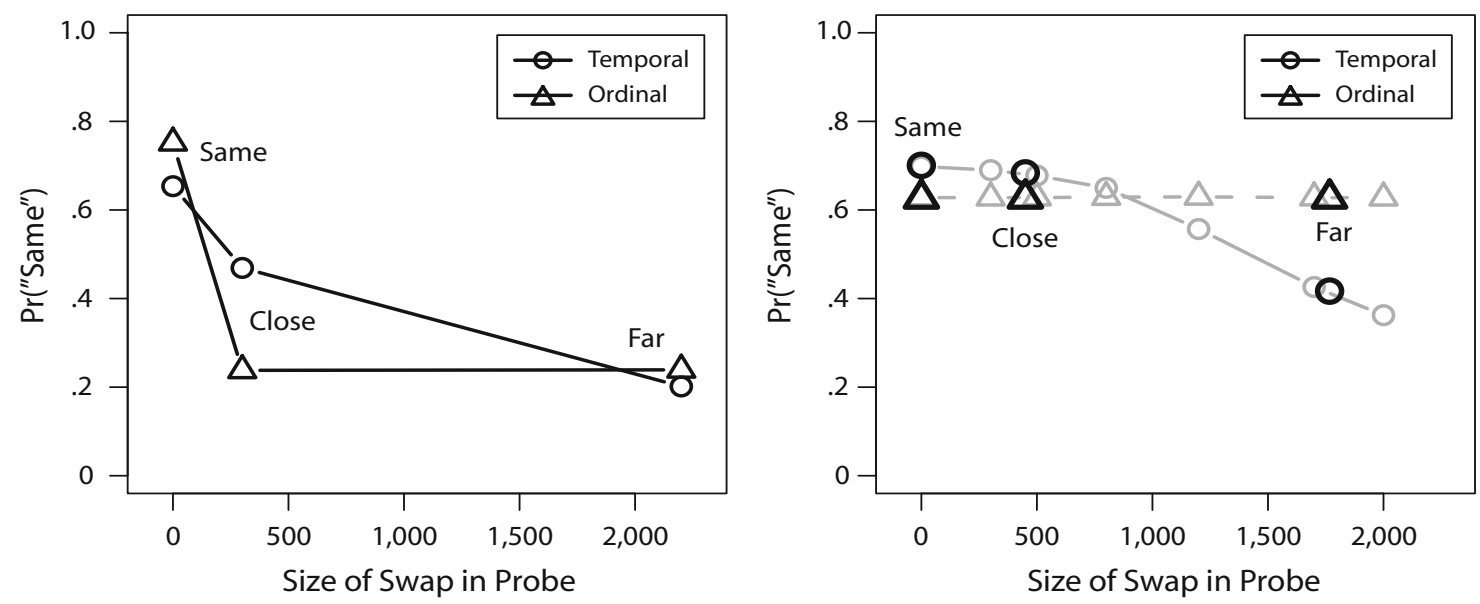

Figure 4. Predicted "same" responses from the time-based model and position-based model for the serial recognition task (left panel) and the temporal recognition task (right panel), calculated from maximum likelihood parameter estimates. As in Figure 2, responses are plotted as a function of the displacement in the probe. Data for the temporal recognition task are grouped according to the displacement in time of the item perturbed in the lure (gray), and also by the condition (black symbols).

be forced into giving little, if any, weight to the temporal dimension (as happened for the fit to Participant 11's data; see the Appendix), which would also result in a predicted null difference between same and close lures. In contrast, the order-based model provides a good account of the data, predicting a drop in "same" responses from same to close probes, but then predicting equivalent response proportions for close and far probes.

Quantitative verification of the relative accounts of the models is given in Table 1, which summarizes the minimized $-\ln L$ s for individual participants (see the Appendix for maximum likelihood parameter estimates). Since the individual participant fits provide independent sources of evidence about the fit of the models, we can sum the $-\ln L$ s (i.e., multiply likelihoods) to calculate a combined $-\ln L$ of all the data under the model. Calculating a likelihood ratio from these sums (by exponentiating the difference between the summed $\ln L s$ for the two models) gives an interpretable metric of the relative fits of the models. The likelihood ratio for the two models is over $2 \times 10^{30}$ in favor of the positional model: The positional model provides a clearly superior account of the data.

In contrast, the time-based model provides a good account of participants' performance on the timing trials. The models' best-fitting predictions are shown in the right panel of Figure 4. The time-based model accounts for the small difference in "same" response proportions between the same and close probes, and the large difference between the close and far probes. The model also captures the flatness in the function relating "same" response proportions to temporal displacement for small displacements, and an accelerating drop for larger displacements. Unsurprisingly, the order-based model, which does not incorporate any temporal information beyond order, is insensitive to temporal displacement, predicting equal response proportions for same, close, and far probes. These impressions are confirmed by inspection of the minimized $-\ln L \mathrm{~s}$ for individual participants given in the right half of Table 1. The likelihood ratio for the temporal recognition task is over $10^{33}$ in favor of the time-based model, providing clear evidence for this model.

Table 1

Minimized Negative Log-Likelihoods for the Time-Based Model and Ordinal Model

\begin{tabular}{|c|c|c|c|c|}
\hline \multirow[b]{2}{*}{ Participant } & \multicolumn{2}{|c|}{ Serial Recognition } & \multicolumn{2}{|c|}{ Temporal Recognition } \\
\hline & Time-Based & Order-Based & Time-Based & Order-Basec \\
\hline 1 & 4.92 & 4.39 & 7.92 & 7.95 \\
\hline 2 & 6.02 & 4.88 & 5.66 & 6.55 \\
\hline 3 & 4.92 & 4.39 & 4.37 & 4.91 \\
\hline 4 & 4.83 & 4.52 & 4.82 & 4.95 \\
\hline 5 & 5.63 & 4.82 & 4.88 & 5.32 \\
\hline 6 & 8.55 & 4.39 & 3.80 & 11.29 \\
\hline 7 & 6.47 & 4.49 & 4.75 & 4.75 \\
\hline 8 & 5.07 & 4.50 & 4.36 & 4.52 \\
\hline 9 & 4.16 & 4.65 & 4.93 & 6.02 \\
\hline 10 & 1.92 & 2.66 & 4.15 & 9.20 \\
\hline 11 & 2.69 & 2.69 & 7.23 & 7.29 \\
\hline 12 & 0.97 & 0.97 & 4.00 & 9.46 \\
\hline 13 & 0.97 & 0.97 & 3.57 & 12.75 \\
\hline 14 & 10.76 & 5.09 & 5.34 & 6.09 \\
\hline 15 & 1.60 & 1.60 & 4.66 & 8.15 \\
\hline 16 & 6.36 & 4.47 & 5.26 & 5.28 \\
\hline 17 & 7.91 & 4.02 & 4.47 & 5.32 \\
\hline 18 & 4.32 & 5.99 & 4.11 & 6.80 \\
\hline 19 & 6.15 & 5.36 & 4.73 & 4.75 \\
\hline 20 & 14.86 & 1.90 & 2.92 & 6.73 \\
\hline 21 & 4.43 & 6.00 & 6.82 & 8.31 \\
\hline 22 & 9.44 & 3.42 & 4.33 & 4.65 \\
\hline 23 & 0 & 0 & 0.95 & 19.02 \\
\hline 24 & 5.95 & 4.31 & 5.27 & 5.39 \\
\hline 25 & 7.29 & 3.59 & 4.37 & 5.63 \\
\hline 26 & 9.54 & 4.06 & 4.28 & 5.45 \\
\hline 27 & 8.36 & 3.92 & 4.47 & 5.32 \\
\hline 28 & 4.82 & 4.82 & 4.48 & 4.58 \\
\hline 29 & 10.78 & 3.32 & 4.36 & 5.76 \\
\hline 30 & 14.06 & 2.89 & 4.03 & 12.81 \\
\hline Sum & 183.75 & 113.08 & 139.29 & 215.00 \\
\hline
\end{tabular}




\section{GENERAL DISCUSSION}

Two conclusions can be drawn from the experiment and simulation results. First, the results provide clear further evidence against models that specify a single temporal representation underlies performance on short-term order and timing tasks. Despite the fact that favorable conditions were provided to find any temporal isolation effects-by using a serial recognition task and thus minimizing dependencies on output order (see Lewandowsky et al., in press), having participants explicitly remember the timing of items, and manipulating timing over a wide range (see Nimmo \& Lewandowsky, 2005) —no TIEs were observed in participants' performance. Furthermore, the simulations showed that although a time-based model could account for participants' memory for the timing of items, the model performed more poorly when accounting for performance on the serial recognition task than a comparable order-based model. However, the order-based model did not provide a satisfactory account of performance on the timing task, implying that participants can draw on temporal or ordinal information when performing the tasks considered here. Furthermore, if such a hybrid temporalordinal theory is accepted, the results imply that attention can be allocated to temporal and positional information at the time of test. The implications of the modeling and the experiment are now considered in more detail.

\section{Modeling Memory for Ordinal and Temporal Information}

The introduction here of a model of serial and temporal recognition makes a novel theoretical contribution to the literature on short-term memory. Although the serial recognition task has been argued to be useful in examining order memory independent of contributions of long-term knowledge (Gathercole, Pickering, Hall, \& Peaker, 2001; Thorn, Gathercole, \& Frankish, 2002), no model to date has been extended to account for serial recognition. Although it has been argued here that the temporal matching model does not provide an adequate quantitative account of serial recognition data, the simulations using a positional version of the model provide a satisfactory initial account of the serial recognition task. Furthermore, as discussed below, models have not generally been concerned with memory for temporal recognition; implementing a model using similar principles (although different types of information) to account for performance on serial recognition and temporal recognition is a step toward unification of the literature on short-term memory for time (e.g., Collier \& Logan, 2000; Crowder \& Greene, 1987; Pich, 2000; Saito, 2001; Watkins et al., 1992) and short-term memory for order (for a review, see Marshuetz, 2005).

One extension to the temporal matching model would be to account for the dynamics of recall as well as response probabilities. Ratcliff (1981) demonstrated that a model of perceptual matching, based on principles similar to those assumed here, ably accounted for changes in observed response probabilities and response latencies in the framework of a diffusion model of reaction time (Ratcliff, 1978). A similar extension of temporal matching model would be advantageous in broadening the scope of the model, as well as providing additional constraints following from the requirement to simultaneously account for accuracy and response time data (see, e.g., Ratcliff \& Smith, 2004). One interesting feature of the serial recognition and temporal recognition tasks is that the presentation of information is extended over time, in contrast to perceptual matching of letter strings. The extension of the presentation of information over time potentially allows for the examination of information accumulation by allowing participants to make a response at any point during the probe sequence: This would allow determination of the extent to which the probe sequence predicts which response is made, and the point during the probe at which the response is made (see, e.g., Ludwig, Gilchrist, McSorley, \& Baddeley, 2005, for an application to saccadic decision making).

\section{Implications for Theories of Short-Term Memory}

The results here complement other results in again failing to demonstrate an effect of temporal isolation on shortterm order memory (Lewandowsky \& Brown, 2005; Lewandowsky et al., 2006; Nimmo \& Lewandowsky, 2005, 2006). This is problematic for theories which assume that a sole temporal dimension underlies memory for both the order of items and their timing. An alternative to pure time-based models is the class of models referred to as event-based models (Lewandowsky \& Brown, 2005). In such models order is not represented by time but by some other representation or process. Models such as the startend model (Henson, 1998) assume, like the time-driven OSCAR model (Brown et al., 2000), that items are associated with positional markers; an important contrast with OSCAR is that the positional markers in these models are not time-based, but code for ordinal position. Similarly, in models such as the serial-order-in-a-box model (Farrell, 2006; Farrell \& Lewandowsky, 2002) and the primacy model (Page \& Norris, 1998), the order of items is represented by their relative activation. In these models, like the positional matching model here, temporal isolation is not expected to have an effect on recall accuracy.

However, the dissociation between timing and order models indicates that event-based models of short-term memory are incomplete; although these models can account for serial recall performance, the demonstration that a position-based model provides a poor account of memory for the timing of items indicates that a comprehensive model of sequence memory will require multiple forms of representation. This conclusion is consistent with the finding of Lewandowsky et al. (in press) that TIEs may be witnessed even in order memory when output order is unconstrained. Lewandowsky et al. (in press) explained their results with reference to the hybrid SIMPLE model, which incorporates both temporal and positional dimensions (Brown et al., 2006; Brown et al., 2007). The results presented here are consistent with the suggestion that participants attending primarily to an ordinal dimension when performing serial recognition, and attending primarily to a temporal dimension when performing temporal recognition. Given that the task was postcued after stimulus pre- 
sentation, this implies that the trade-off between temporal and positional information is not due to differential encoding of the different types of information at study, but rather follows from attentional weighting at retrieval; such a conclusion was also reached by Lewandowsky et al. (in press) from their postcuing procedure in their consideration of the SIMPLE distinctiveness model. Generally, this conclusion is consistent with exemplar models of categorization, the forebears of SIMPLE, in which attention is given to perceptual or psychological dimensions at the time of categorization (i.e., retrieval; Kruschke, 1992; Nosofsky, 1986).

However, some caution is warranted in relating the timing information implied here to that assumed in models such as SIMPLE. It is not necessarily the case that the temporal information implicated in tasks requiring the recall of timing information such as that used here (see also Collier \& Logan, 2000; Crowder \& Greene, 1987; Elvevåg et al., 2004; Glenberg \& Jona, 1991; Saito, 2001; Watkins et al., 1992 ) is the same temporal information assumed in models such as SIMPLE and OSCAR. Indeed, one possibility is that, in timing tasks, participants are not remembering items along a single temporal dimension, but are instead remembering the durations or intervals between items. This suggests an explanation for Saito's (2001) finding of a correlation across individuals between digit span and a rhythm memory task, which appears to be inconsistent with the assumption of independent timing and order dimensions. This explanation is that the order of items is represented as in event-based theories (Farrell \& Lewandowsky, 2002; Page $\&$ Norris, 1998), but that durations or intervals form part of the representation of items. Thus, correlations between timing and order tasks may be introduced through commonalities in the ordering mechanism, but with participants nonetheless able to use ordering mechanisms as specified in event-based theories to recall item information such as timing. However, this would leave unexplained Elvevåg et al. (2004)'s finding of deficits in schizophrenia patients in both probed recall and duration identification, where in the latter task only a single stimulus was presented on each trial, removing any demands on order memory. What is clear is that resolving these issues through further investigation of the relationship between memory for timing and memory for order will advance theory in short-term memory generally (Crowder \& Greene, 1987; Watkins et al., 1992).

\section{AUTHOR NOTE}

The experiment reported in this article was run as part of the second author's undergraduate dissertation. This research was presented at the 4th International Conference on Memory (July 2006) in Sydney, Australia. We thank James Melhorn for recording the stimuli used in the experiments and Fiona Laver, Ian Neath, Simon Dennis, and two anonymous reviewers for their comments. Correspondence should be addressed to S. Farrell, Department of Psychology, University of Bristol, 12a Priory Road, Clifton, Bristol BS8 1TU, England (e-mail: simon.farrell@bristol.ac.uk).

\section{REFERENCES}

BAdDeley, A. D. (1986). Working memory. New York: Oxford University Press.

Bakeman, R., \& McArthur, D. (1996). Picturing repeated measures: Comments on Loftus, Morrison, and others. Behavior Research Methods, Instruments, \& Computers, 28, 584-589.
BJork, R. A., \& Whitten, W. B. (1974). Recency-sensitive retrieval processes in long-term free recall. Cognitive Psychology, 6, 173-189.

Boersma, P., \& Weenink, D. (2006). Praat: Doing phonetics by computer (Version 4.4.26) [Computer software]. Retrieved 3rd August, 2006, from www.praat.org/.

Brainard, D. H. (1997). The Psychophysics Toolbox. Spatial Vision, 10, 433-436.

Brown, G. D. A., Morin, C., \& Lewandowsky, S. (2006). Evidence for time-based models of free recall. Psychonomic Bulletin \& Review, 13, 717-723.

Brown, G. D. A., Neath, I., \& Chater, N. (2007). A temporal ratio model of memory. Psychological Review, 114, 539-576.

Brown, G. D. A., Preece, T., \& Hulme, C. (2000). Oscillator-based memory for serial order. Psychological Review, 107, 127-181.

Burgess, N., \& Hitch, G. J. (1999). Memory for serial order: A network model of the phonological loop and its timing. Psychological Review, 106, 551-581.

Collier, G. L., \& Logan, G. (2000). Modality differences in short-term memory for rhythms. Memory \& Cognition, 28, 529-538.

Crowder, R. G., \& Greene, R. L. (1987). On the remembrance of times past: The irregular list technique. Journal of Experimental Psychology: General, 116, 265-278.

DeCarlo, L. T. (2003). Using the PLUM procedure of SPSS to fit unequal variance and generalized signal detection models. Behavior Research Methods, Instruments, \& Computers, 35, 49-56.

Elvevåg, B., Brown, G. D. A., McCormack, T., Vousden, J. I., \& GolDBERG, T. E. (2004). Identification of tone duration, line length, and letter position: An experimental approach to timing and working memory deficits in schizophrenia. Journal of Abnormal Psychology, 113, 509-521.

Estes, W. K. (1997). Processes of memory loss, recovery, and distortion. Psychological Review, 104, 148-169.

FARRELL, S. (2006). Mixed-list phonological similarity effects in delayed serial recall. Journal of Memory \& Language, 55, 587-600.

FARRELL, S., \& LEWANDOWSKY, S. (2002). An endogenous distributed model of ordering in serial recall. Psychonomic Bulletin \& Review, 9, 59-79.

FleXser, A. J., \& Bower, G. H. (1974). How frequency affects recency judgments: A model for recency discrimination. Journal of Experimental Psychology, 103, 706-716.

Gallistel, C. R., \& GibBon, J. (2000). Time, rate, and conditioning. Psychological Review, 107, 289-344.

Gathercole, S. E., Pickering, S. J., Hall, M., \& Peaker, S. M. (2001). Dissociable lexical and phonological influences on serial recognition and serial recall. Quarterly Journal of Experimental Psychology, 54A, 1-30.

Gibbon, J., \& Church, R. M. (1990). Representation of time. Cognition, 37, 23-54.

GlenberG, A. M. (1987). Temporal context and memory. In D. S. Gorfein \& R. R. Hoffman (Eds.), Memory and learning: The Ebbinghaus Centennial Conference (pp. 173-190). Hillsdale, NJ: Erlbaum.

Glenberg, A. M., \& Jona, M. (1991). Temporal coding in rhythm tasks revealed by modality effects. Memory \& Cognition, 19, 514-522.

Glenberg, A. M., Mann, S., Altman, L., Forman, T., \& Procise, S. (1989). Modality effects in the coding and reproduction of rhythms. Memory \& Cognition, 17, 373-383.

Glenberg, A. M., \& Swanson, N. G. (1986). A temporal distinctiveness theory of recency and modality effects. Journal of Experimental Psychology: Learning, Memory, \& Cognition, 12, 3-15.

Henson, R. N. A. (1998). Short-term memory for serial order: The startend model. Cognitive Psychology, 36, 73-137.

Kruschke, J. K. (1992). ALCOVE: An exemplar-based connectionist model of category learning. Psychological Review, 99, 22-44.

LeE, C. L., \& Estes, W. K. (1977). Order and position in primary memory for letter strings. Journal of Verbal Learning \& Verbal Behavior, 16, 395-418.

Lewandowsky, S., \& Brown, G. D. A. (2005). Serial recall and presentation schedule: A micro-analysis of local distinctiveness. Memory, 13, 283-292.

Lewandowsky, S., Brown, G. D. A., Wright, T., \& Nimmo, L. M. (2006). Timeless memory: Evidence against temporal distinctiveness models of short-term memory for serial order. Journal of Memory \& Language, 54, 20-38. 
Lewandowsky, S., \& Murdock, B. B. (1989). Memory for serial order. Psychological Review, 96, 25-57.

Lewandowsky, S., Nimmo, L. M., \& Brown, G. D. A. (in press). When temporal isolation benefits memory for serial order. Journal of Memory \& Language.

Ludwig, C., Gilchrist, I. D., McSorley, E., \& Baddeley, R. J. (2005). The temporal impulse response underlying saccadic decisions. Journal of Neuroscience, 25, 9907-9912.

MARSHUETZ, C. (2005). Order information in working memory: An integrative review of evidence from brain and behavior. Psychological Bulletin, 131, 323-339.

Murdock, B. B. (1995). Developing TODAM: three models for serialorder information. Memory \& Cognition, 23, 631-645.

Neath, I. (1993). Contextual and distinctive processes and the serial position function. Journal of Memory \& Language, 32, 820-840.

NEATH, I. (1999). Modelling the disruptive effects of irrelevant speech on order information. International Journal of Psychology, 34, 410418.

Neath, I., \& Brown, G. D. A. (2006). Further applications of a local distinctiveness model of memory. In B. H. Ross (Ed.), The psychology of learning and motivation (Vol. 46, pp. 201-243). San Diego: Academic Press.

Neath, I., \& Crowder, R. G. (1990). Schedules of presentation and temporal distinctiveness in human memory. Journal of Experimental Psychology: Learning, Memory, \& Cognition, 16, 316-327.

Nelder, J. A., \& Mead, R. (1965). A simplex method for function minimization. Computer Journal, 7, 308-313.

Nicholls, A. P., \& Jones, D. M. (2002). Capturing the suffix: Cognitive streaming in immediate serial recall. Journal of Experimental Psychology: Learning, Memory, \& Cognition, 28, 12-28.

Nimmo, L. M., \& Lewandowsky, S. (2005). From brief gaps to very long pauses: Temporal isolation does not benefit serial recall. Psychonomic Bulletin \& Review, 12, 999-1004.

Nimmo, L. M., \& LeWANDOWsKy, S. (2006). Distinctiveness revisited: Unpredictable temporal isolation does not benefit short-term serial recall of heard or seen events. Memory \& Cognition, 34, 1368-1375.

Nosofsky, R. M. (1986). Attention, similarity, and the identificationcategorization relationship. Journal of Experimental Psychology: Learning, Memory, \& Cognition, 115, 39-61.

PAGE, M. P. A., \& Norris, D. (1998). The primacy model: A new model of immediate serial recall. Psychological Review, 105, 761-781.

Parmentier, F. B. R., King, S., \& Dennis, I. (2006). Local temporal distinctiveness does not benefit auditory verbal and spatial serial recall. Psychonomic Bulletin \& Review, 13, 458-465.

Pelli, D. G. (1997). The Video Toolbox software for visual psychophysics: Transforming numbers into movies. Spatial Vision, 10, 437-442.

PICH, J. (2000). The role of subvocalization in rehearsal and maintenance of rhythmic patterns. Spanish Journal of Psychology, 3, 63-67.

Pinheiro, J. C., \& Bates, D. M. (2000). Mixed-effects models in $S$ and S-Plus. New York: Springer.

Ratcliff, R. (1978). A theory of memory retrieval. Psychological Review, 85, 59-108.

RATCLIFF, R. (1981). A theory of order relations in perceptual matching. Psychological Review, 88, 552-572.
RAtclifF, R., \& SMith, P. L. (2004). A comparison of sequential sampling models for two-choice reaction time. Psychological Review, 111, 333-367.

Ross, J., \& Houtsma, A. J. M. (1994). Discrimination of auditory temporal patterns. Perception \& Psychophysics, 56, 19-26.

Rouder, J. N., \& LU, J. (2005). An introduction to bayesian hierarchical models with an application in the theory of signal detection. Psychonomic Bulletin \& Review, 12, 573-604.

RYAN, J. (1969). Temporal grouping, rehearsal and short-term memory. Quarterly Journal of Experimental Psychology, 21, 148-155.

SaITo, S. (2001). The phonological loop and memory for rhythms: An individual differences approach. Memory, 9, 313-322.

Schab, F. R., \& Crowder, R. G. (1989). Accuracy of temporal coding: Auditory-visual comparisons. Memory \& Cognition, 17, 384-397.

Scott, S. K. (1998). The point of P-centres. Psychological Research, 61, 4-11.

Staddon, J. E. R., \& Higa, J. J. (1999). Time and memory: Towards a pacemaker-free theory of interval timing. Journal of the Experimental Analysis of Behavior, 1, 215-251.

Thorn, A. S. C., Gathercole, S. E., \& Frankish, C. R. (2002). Language familiarity effects in short-term memory: The role of output delay and long-term knowledge. Quarterly Journal of Experimental Psychology, 55A, 1363-1383.

Watkins, M. J., LeCompte, D. C., Elliott, M. N., \& Fish, S. B. (1992). Short-term memory for the timing of auditory and visual signals. Journal of Experimental Psychology: Learning, Memory, \& Cognition, 18, 931-937.

WeARDEN, J. H. (2002). Traveling in time: A time-left analogue for humans. Journal of Experimental Psychology: Animal Behavior Processes, 28, 200-208.

Wearden, J. H., \& Doherty, M. F. (1995). Exploring and developing a connectionist model of animal timing: Peak procedure and fixedinterval simulations. Journal of Experimental Psychology: Animal Behavior Processes, 21, 99-115.

\section{NOTES}

1. Another class of serial order memory models in which time has effects on recall are models in which forgetting is due to time-related decay (e.g., Baddeley, 1986; Page \& Norris, 1998). The term time-based here does not cover decay-based models, and is used to refer only to models in which ordinal confusions are due to temporal proximity of items (i.e., temporal context models and temporal distinctiveness models).

2. Mauchly's test for sphericity was significant $(p<.001)$; ANOVA results are reported with Greenhouse-Geisser correction.

3 . Although the assumption of linearity has precedents in the literature (Gibbon \& Church, 1990; Wearden, 2002), some theorists have argued that memory for the time of events is logarithmically compressed (Brown et al., 2007). As well as introducing an additional parameter (the base of the logarithm), logarithmic compression would mean that the study list and probe sequence would be on different scales; accordingly, some mechanism would need to be specified by which the representation of the study list could be reliably decompressed to allow comparison with the probe list. 
APPENDIX

Maximum Likelihood Parameter Estimates for Individual Participants for the Time-Based and Position-Based Models

\begin{tabular}{|c|c|c|c|c|c|c|c|c|}
\hline \multirow[b]{3}{*}{ Participant } & \multicolumn{4}{|c|}{ Serial Recognition } & \multicolumn{4}{|c|}{ Temporal Recognition } \\
\hline & \multicolumn{2}{|c|}{ Time-Based } & \multicolumn{2}{|c|}{ Order-Based } & \multicolumn{2}{|c|}{ Time-Based } & \multicolumn{2}{|c|}{ Order-Based } \\
\hline & $w_{\mathrm{D}}$ & $\mathrm{C}_{\mathrm{S}}$ & $w_{\mathrm{D}}$ & $\mathrm{C}_{\mathrm{T}}$ & $w_{\mathrm{D}}$ & $\mathrm{C}_{\mathrm{S}}$ & $w_{\mathrm{D}}$ & $\mathrm{C}_{\mathrm{T}}$ \\
\hline 1 & 3.35 & 3.92 & 5.90 & 4.29 & 1.09 & 3.70 & 7.17 & 3.84 \\
\hline 2 & 5.63 & 5.70 & 11.67 & 7.50 & 8.99 & 5.62 & 0.54 & 6.11 \\
\hline 3 & 3.35 & 3.92 & 5.90 & 4.29 & 7.58 & 5.10 & 1.08 & 5.01 \\
\hline 4 & 2.93 & 4.19 & 5.10 & 4.48 & 4.53 & 3.14 & 17.48 & 3.98 \\
\hline 5 & 1.13 & 4.92 & 4.65 & 5.01 & 5.56 & 2.75 & 12.82 & 3.29 \\
\hline 6 & 3.07 & 3.53 & 8.19 & 4.32 & 19.22 & 6.89 & 5.01 & 4.24 \\
\hline 7 & 4.97 & 5.16 & 10.31 & 6.59 & 1.07 & 4.06 & 0.51 & 4.99 \\
\hline 8 & 3.19 & 4.37 & 5.92 & 4.80 & 5.72 & 4.38 & 13.48 & 5.02 \\
\hline 9 & 3.86 & 3.64 & 5.54 & 3.86 & 7.64 & 3.41 & 18.42 & 3.62 \\
\hline 10 & 34.91 & 8.83 & 18.80 & 8.84 & 14.81 & 5.67 & 0.53 & 5.13 \\
\hline 11 & 0.10 & 10.22 & 0.24 & 10.04 & 1.41 & 5.78 & 1.11 & 6.05 \\
\hline 12 & 53.08 & 8.83 & 37.18 & 8.84 & 15.52 & 4.97 & 4.56 & 3.21 \\
\hline 13 & 53.08 & 8.83 & 37.18 & 8.84 & 21.72 & 6.63 & 1.12 & 4.36 \\
\hline 14 & 2.65 & 4.37 & 9.69 & 6.03 & 6.89 & 4.09 & 6.74 & 3.89 \\
\hline 15 & 45.80 & 5.60 & 23.06 & 5.63 & 13.25 & 6.23 & 1.08 & 5.01 \\
\hline 16 & 2.57 & 4.36 & 6.69 & 5.12 & 3.45 & 3.59 & 0.53 & 5.13 \\
\hline 17 & 3.45 & 3.93 & 8.57 & 4.85 & 7.48 & 4.20 & 6.74 & 3.89 \\
\hline 18 & 4.00 & 4.53 & 4.50 & 4.48 & 11.97 & 5.81 & 5.01 & 4.24 \\
\hline 19 & 0.17 & 4.93 & 4.70 & 4.27 & 2.26 & 3.26 & 0.51 & 4.99 \\
\hline 20 & 6.85 & 5.42 & 44.74 & 22.43 & 19.85 & 9.32 & 0.65 & 7.36 \\
\hline 21 & 5.63 & 4.98 & 8.26 & 5.57 & 8.40 & 4.24 & 7.17 & 3.84 \\
\hline 22 & 5.68 & 4.56 & 11.98 & 6.08 & 6.62 & 4.74 & 5.01 & 4.24 \\
\hline 23 & 132.81 & 27.95 & 90.05 & 20.94 & 220.12 & 45.91 & 9.10 & 5.48 \\
\hline 24 & 4.14 & 4.46 & 7.98 & 5.18 & 4.71 & 3.49 & 7.17 & 3.84 \\
\hline 25 & 4.90 & 4.01 & 9.52 & 4.89 & 9.37 & 5.30 & 5.01 & 4.24 \\
\hline 26 & 4.03 & 4.84 & 10.87 & 6.57 & 9.22 & 5.26 & 5.01 & 4.24 \\
\hline 27 & 4.38 & 4.59 & 10.39 & 6.05 & 8.27 & 4.90 & 13.48 & 5.02 \\
\hline 28 & 0.30 & 5.06 & 0.23 & 5.16 & 4.11 & 2.54 & 12.86 & 3.40 \\
\hline 29 & 6.79 & 5.41 & 16.66 & 8.84 & 8.95 & 4.75 & 0.53 & 5.13 \\
\hline 30 & 6.11 & 4.76 & 16.13 & 7.44 & 20.78 & 6.57 & 1.12 & 4.36 \\
\hline
\end{tabular}

(Manuscript received August 14, 2006;

revision accepted for publication January 15, 2007.) 